\title{
Accumulation Index using Lambda z
}

National Cancer Institute

\section{Source}

National Cancer Institute. Accumulation Index using Lambda z. NCI Thesaurus. Code C114234.

The predicted accumulation ratio for area under the curve (AUC) calculated using the Lambda z estimated from single dose data. 